\title{
Cellular self-organization on micro-structured surfaces $\uparrow$
}

\begin{abstract}
Peter J. F. Röttgermann, Alicia Piera Alberola and Joachim O. Rädler*
Micro-patterned surfaces are frequently used in high-throughput single-cell studies, as they allow one to image isolated cells in defined geometries. Commonly, cells are seeded in excess onto the entire chip, and non-adherent cells are removed from the unpatterned sectors by rinsing. Here, we report on the phenomenon of cellular self-organization, which allows for autonomous positioning of cells on micropatterned surfaces over time. We prepared substrates with a regular lattice of protein-coated adhesion sites surrounded by PLL-g-PEG passivated areas, and studied the time course of cell ordering. After seeding, cells randomly migrate over the passivated surface until they find and permanently attach to adhesion sites. Efficient cellular self-organization was observed for three commonly used cell lines (HuH7, A549, and MDA-MB-436), with occupancy levels typically reaching $40-60 \%$ after $3-5 \mathrm{~h}$. The time required for sorting was found to increase with increasing distance between adhesion sites, and is well described by the time-to-capture in a random-search model. Our approach thus paves the way for automated filling of cell arrays, enabling high-throughput single-cell analysis of cell samples without losses.
\end{abstract}

Received 13th September 2013 Accepted 23rd December 2013

DOI: $10.1039 / \mathrm{c} 3 \mathrm{sm} 52419 a$

www.rsc.org/softmatter throughput ${ }^{22,23}$ and low viability rates, as cells can be ruptured due to shear forces. ${ }^{\mathbf{2 0} 24}$ All these techniques try to achieve a higher occupancy rate than that given by the inherently stochastic process of sedimentation. The number of trapped cells per adhesive spot follows a Poisson distribution..$^{25-28}$ As a result, such arrays exhibit a broad distribution of cell numbers on the adhesive islands. For an expected value of $\lambda=1$ (one cell per site), the maximal single-cell occupancy achievable is $37 \%$.

In this article, we present a novel approach to the generation of single-cell arrays, utilizing cellular self-organization. Cells are seeded onto substrates patterned with protein-coated islands and separated by PLL- $g$-PEG passivated zones. The cells migrate to the passivated areas and spread onto the protein-coated sites upon contact. Thus, cells are sorted according to surface affinity and the population undergoes a transition from a spatially random distribution to an ordered array. Here, the phenomenon of cellular self-organization on micro-structured surfaces is systematically addressed. We quantify the time courses of occupancy levels using automated cell tracking. In addition, the correlation between lattice distance (distance between the centres of neighbouring sites) and filling rate is analysed, and the influence of cell motility is studied for three different cell lines (A549, HuH7, and MDA-MB436). Finally, we describe the time-to-order onto the lattice sites by a simple model adapted from the physics of chemoreception.

\section{Materials and methods}

\section{Cell culture}

The A549 cell line (ATCC) was cultured in modified Eagle's medium with Earle's salts (c-c-pro) and $4 \mathrm{mM}$ L-glutamine, the
80539 Munich, Germany.E-mail: peter.roettgermann@physik.lmu.de; raedler@lmu. de; Fax: +49892180 3182; Tel: +498921802438

$\uparrow$ Electronic supplementary information (ESI) available. See DOI: $10.1039 / \mathrm{c} 3 \operatorname{sm} 52419 \mathrm{a}$ 
HuH7 cell line (I.A.Z Toni Lindl GmbH) was cultured in Dulbecco's modified Eagle's medium with $4.5 \mathrm{~g} \mathrm{~L}^{-1}$ glucose, 110 $\mathrm{mg} \mathrm{\textrm {L } ^ { - 1 }}$ sodium pyruvate (c-c-pro) and $2 \mathrm{mM}$ L-glutamine, and MDA-MB-436 (ATCC) cells were grown in modified Eagle's medium and Ham's F12 (c-c-pro) with $2.5 \mathrm{mM}$ L-glutamine. All cell media were supplemented with $10 \%$ fetal calf serum (FCS). The cells were cultured at $37{ }^{\circ} \mathrm{C}$, with $5 \% \mathrm{CO}_{2}$ in an incubator. Cells were grown to 70-80\% confluence, trypsinized (A549 and $\mathrm{HuH7}$ ) or scraped off (MDA-MB-436) and centrifuged at 1000 rpm for $3 \mathrm{~min}$. Cell pellets were resuspended in either cell medium or, for the experiments, in Leibovitz's L15 medium with GlutaMAX (Gibco) and 10\% FCS to provide optimal growth conditions. The seeding density was determined using a Neubauer counting chamber. To track nuclei, cells were stained with the permeable fluorescent dye Hoechst 33342 (Life Technologies) by adding $25 \mathrm{nM}$ dye to the suspension (incubation at $37{ }^{\circ} \mathrm{C}$ for $20 \mathrm{~min}$ ).

\section{Surface patterning and sample preparation}

The micro-structured surfaces were produced by selective oxygen plasma treatment (Femto Diener, $40 \mathrm{~W}$ for $3 \mathrm{~min}$ ) on a Topas substrate ( $\mu$-dishes ibidi $\mathrm{GmbH}$ ) with subsequent passivation. Selectivity was achieved using a polydimethylsiloxane (PDMS) stamp (cast from a master produced by photolithography) as a mask. The parts exposed to plasma were passivated with PLL(20k)-g(3.5)-PEG(2k) (SuSoS AG) at 1 $\mathrm{mg} \mathrm{ml} \mathrm{m}^{-1}$ in aqueous buffer $(10 \mathrm{mM}$ HEPES $\mathrm{pH} 7.4$ and 150 $\mathrm{mM} \mathrm{NaCl}$ ). The remaining sectors were rendered cell adherent by exposure to $50 \mu \mathrm{g} \mathrm{ml}^{-1}$ fibronectin (YoProteins) for $1 \mathrm{~h}$. The probes were thoroughly rinsed with PBS; before cell seeding the samples were stored in cell medium at room temperature.

PLL-g-PEG surfaces without patterns were treated in the same way. $30 \mu \mathrm{m} \times 30 \mu \mathrm{m}$ square adhesion sites were used, as this size turned out to be reasonable for single-cell adhesion of all three cell lines. Cells were seeded to achieve a filling of roughly one cell per adhesion site (e.g. for a lattice distance of $90 \mu \mathrm{m}, 65000$ cells were seeded into a $\mu$-dish). The micropattern shown in Fig. 1B was formed using a mixture of $20 \mu \mathrm{g}$ $\mathrm{ml}^{-1}$ fibronectin and $30 \mu \mathrm{g} \mathrm{ml}{ }^{-1}$ fibrinogen conjugated with Alexa Fluor 488 (Life Technologies).

\section{Time-resolved fluorescence microscopy}

Images were taken with an iMIC digital microscope with phase contrast (TILL Photonics) and a Zeiss objective $(10 \times$ Ph1 0.3 EC Plan Neofluar). Samples were kept at a constant temperature of $37^{\circ} \mathrm{C}$ with an ibidi heating system (ibidi GmbH). Fluorescently labelled nuclei were imaged with an oligochrome light source (TILL Photonics) in combination with a single-band long-pass DAPI HC filter set (AHF Analysetechnik). Pictures were recorded every $10 \mathrm{~min}$ over a period of $24 \mathrm{~h}$.

\section{Image and data analysis}

Raw images were pre-processed in ImageJ: the background was subtracted with a built-in function. To highlight the nuclei, a band-pass filter was applied. Images were binarized by setting a
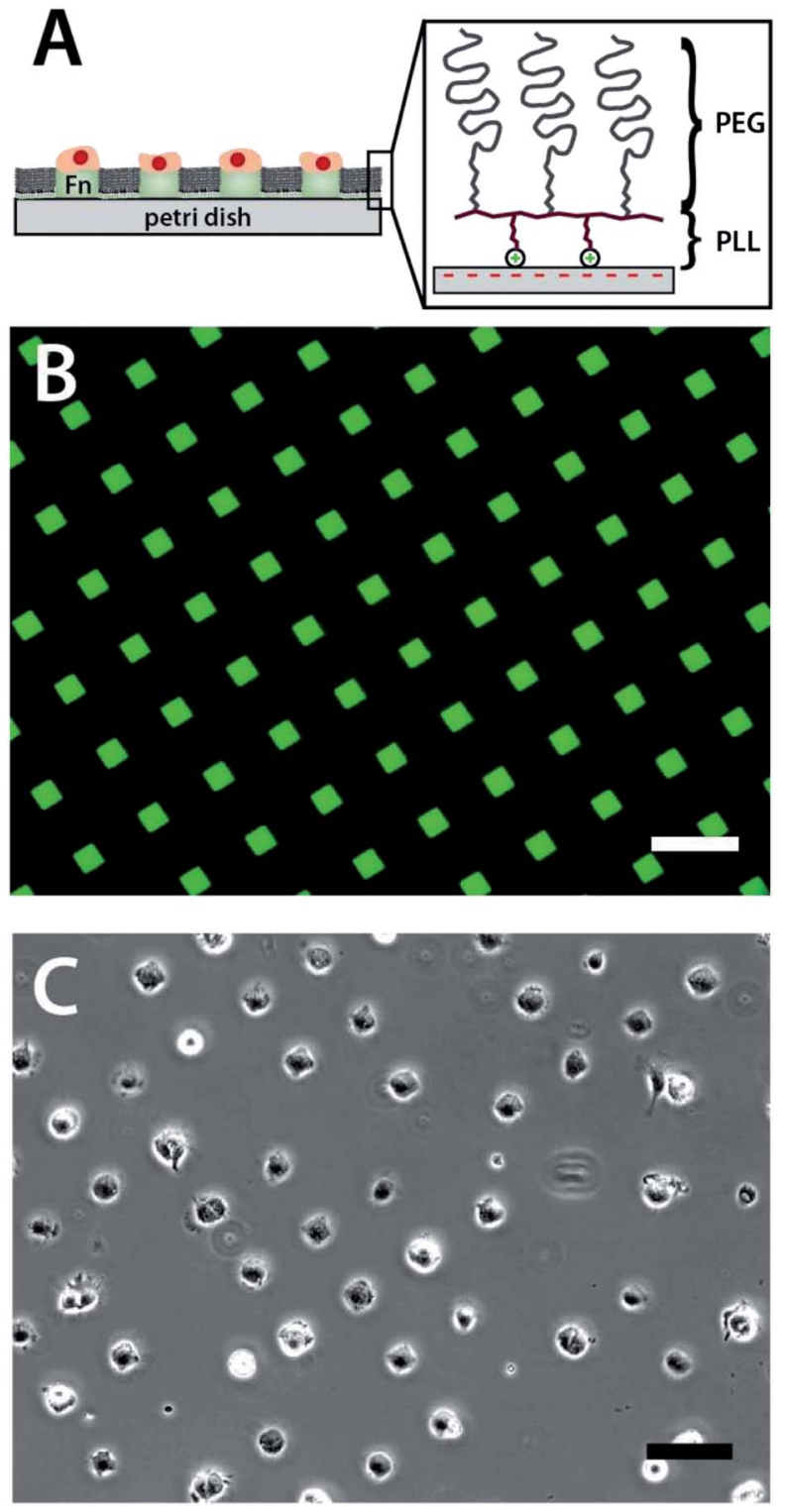

Fig. 1 Patterning technique. (A) A micro-structured surface was created by plasma-induced patterning with a backfill on a Topas surface. Plasma-treated areas were incubated with PLL(2Ok)-g(3.5)PEG(2k) (A). PEG-free areas were coated with fibronectin (Fn, green). (B) Homogeneous square pattern of fibronectin and labelled Alexa Fluor 488 fibrinogen. (C) After seeding, cells arrange themselves onto the protein-coated islands, obviating the need for any washing steps. Scale bars: $100 \mu \mathrm{m}$.

global threshold. Binarized images were clustered and tracked with a cell evaluator. ${ }^{29}$ Coordinates of the centre of mass and the area of nuclei were extracted and analysed in MATLAB. The position of the micro-structured lattice was evaluated from the bright-field images for every marker position. Every experiment was repeated at least twice. 20 to 50 image positions were evaluated for each distance and cell line. The mean of all positions was used for the occupancy statistics. The filling factor in Fig. 3B was normalized to the cell density to compare the different lattice distances. 


\section{Cell motility analysis}

The mean square displacement was calculated as

$$
\operatorname{MSD}(t)=\left\langle\left\langle\left(\boldsymbol{X}_{i}\left(t+t_{0}\right)-X_{i}\left(t_{0}\right)\right)^{2}\right\rangle_{t_{0}}\right\rangle_{i}
$$

where $\boldsymbol{X}_{i}(t)$ denotes the centre of cell $i$ at time $t .\langle\cdot\rangle_{t_{0}}$ designates the mean of the reference frame $t_{0}$ over all times $t \cdot\langle\cdot\rangle_{i}$ is the average over all tracked cells. Dead cells, as well as nuclei exceeding a maximal area (indication of two fused cells), were excluded from analysis. For every cell line at least 100 tracks were analysed. Cell motility was defined by the effective diffusion constant $D$ in two dimensions defined as

$$
\operatorname{MSD}(t)=4 D t
$$

The standard deviation was calculated from the cell motility of different fitted endpoints.

\section{Poisson distribution analysis}

The Poisson distribution is stated as:

$$
P(k)=\frac{\lambda^{k}}{k !} e^{-\lambda}
$$

where $k$ is the occupation number $(k=0, \ldots, 4)$ and $\lambda$ the expected value. The initial number of cells in one image position was counted. Division of this number by a mesh-grid of 45 $\times 45 \mu \mathrm{m}$ (adhesion site + cell radius) defines the expected value $\lambda$. The added deviation from the calculated distribution was averaged over all measured positions.

\section{Results and discussion}

\section{Cell migration on micro-structured surfaces}

We generated patterns of fibronectin-coated square adhesion sites $(30 \mu \mathrm{m} \times 30 \mu \mathrm{m})$ and passivated PLL- $g$-PEG spacings on a Petri dish as described in the Materials and methods Section and schematically shown in Fig. 1A. The coating procedure described here yields an essentially homogeneous protein distribution, as revealed by the homogeneity of the fluorescent spots obtained using an Alexa 488-labelled fibrinogen coating as can be seen in Fig. 1B. Cells were seeded onto micro-patterned sites with various lattice spacings, and time-lapse series were recorded using fluorescence and phase-contrast microscopy. After 3-5 h, and without any washing steps, a regular single-cell array with a negligible number of misplaced cells was obtained (Fig. 1C).

To illustrate the self-organization process we describe it step by step for the adenocarcinoma cell line A549 as a representative example (see also Movie $\mathrm{S} 7 \dagger$ ): the sedimentation process after seeding takes about $20 \mathrm{~min}$, and the cells are randomly distributed at this stage (Fig. 2A, $0 \mathrm{~h}$ ). Cells that initially settle on PLL-g-PEG passivated areas begin to migrate in a random manner (Fig. 2B, 0-210 min). When a cell makes contact with the edge of an adhesion site (Fig. 2B, $210 \mathrm{~min}$ ), it spreads onto it and fills the entire adhesion area within $30 \mathrm{~min}$ (Fig. 2B, 220$250 \mathrm{~min}$ ). In the absence of perturbations, such as cell division events or intruding cells, cells remain captive on the adhesion sites (Fig. 2B, $310 \mathrm{~min}$ ). With time, more and more adhesion sites are occupied (Fig. 2A, 2-24 h). Ideally, each cell would find a free adhesion site and all sites would eventually be singly occupied. Experimentally, we find certain deviations from this ideal scenario: multiple occupancies, unoccupied sites (vacancies) and interstitial cells. Multiple occupancies can be caused if a single cell, already occupying an adhesion site, divides or different cells arrive at the same adhesion site. We observe that multiple occupancies can be self-corrected by the cells if the lattice distance is less than the cells' specific reaching distance, which is defined as the maximal distance to which cells can extend the filopodia without leaving their adhesion site (Fig. S1A $\dagger$ ). If the next available site lies beyond this distance, the defect cannot be self-corrected and cells are likely to stay on the same site (Fig. S1C $\dagger$ ). We observe up to four cells occupying a single site. The reduced accessibility to the proteins on the substrate causes such cells to increase in height. Other rare events are described in the ESI. $\dagger$

\section{Quantifying cellular self-organization via order parameters}

In order to assess the dependence of the ordering process on elapsed time, we quantify the occupancy level. The fraction of maximally occupied sites depends on the initial number of cells per area and the lattice spacing. In the following, the initial cell density is roughly adjusted to the number of available sites. The lattice distance $d_{1}$ is varied from $70 \mu \mathrm{m}$ to $230 \mu \mathrm{m}$. If a cell is positioned on a site, this site is identified as "filled", as illustrated in Fig. 3A. The occupancy level can now be described by a filling factor $F(t)$, which is the ratio of occupied sites $N_{\mathrm{OC}}$ to the total number of sites $N_{\mathrm{S}}: F(t)=N_{\mathrm{OC}} / N_{\mathrm{S}}$. For the A549 cells, we observed the highest filling rate, about $80 \%$, for $d_{1}=90 \mu \mathrm{m}$ after $24 \mathrm{~h}$ and the poorest occupancy level was obtained for $d_{1}=$ $230 \mu \mathrm{m}(25 \%$ after $24 \mathrm{~h})$. However, the shortest filling time of $15 \mathrm{~h}$ was measured for the smallest distance $d_{1}=70 \mu \mathrm{m}$. For all lattices the initial occupation numbers are in good agreement with a random deposition model as described by a Poisson distribution (eqn (3)). The measured occupancy deviates by only $2-6 \%$ from the theoretically calculated Poisson distribution (Fig. S2†).

In the following, we describe the frequency of array irregularities, i.e. vacancies, interstitial cells and multiple occupations, as they occur during the self-ordering process (Fig. 3A).

Vacancies (unoccupied sites) are accounted for in the filling factor. Cells which have not yet found an adhesion site or have died are defined as interstitial. For the interstitial cells we introduce a defect parameter $I(t)$, which defines the ratio of interstitial cells $N_{\text {IS }}$ to the total number of cells $N_{\mathrm{C}}: I(t)=N_{\mathrm{IS}} / N_{\mathrm{C}}$. As shown in Fig. 3C, where the time course of the interstitial cell number is plotted, the curves saturate at different values: for $d_{1}$ $=70 \mu \mathrm{m}$, only $10 \%$ of the cells are misplaced after $24 \mathrm{~h}$. For larger lattice distances, the ratio of misplaced cells increases drastically, reaching $80 \%$ for $d_{1}=230 \mu \mathrm{m}$. In addition, the number of dead cells strongly increases with the lattice size, as cells that do not find an adhesion site within a day have a low survival rate. 
A
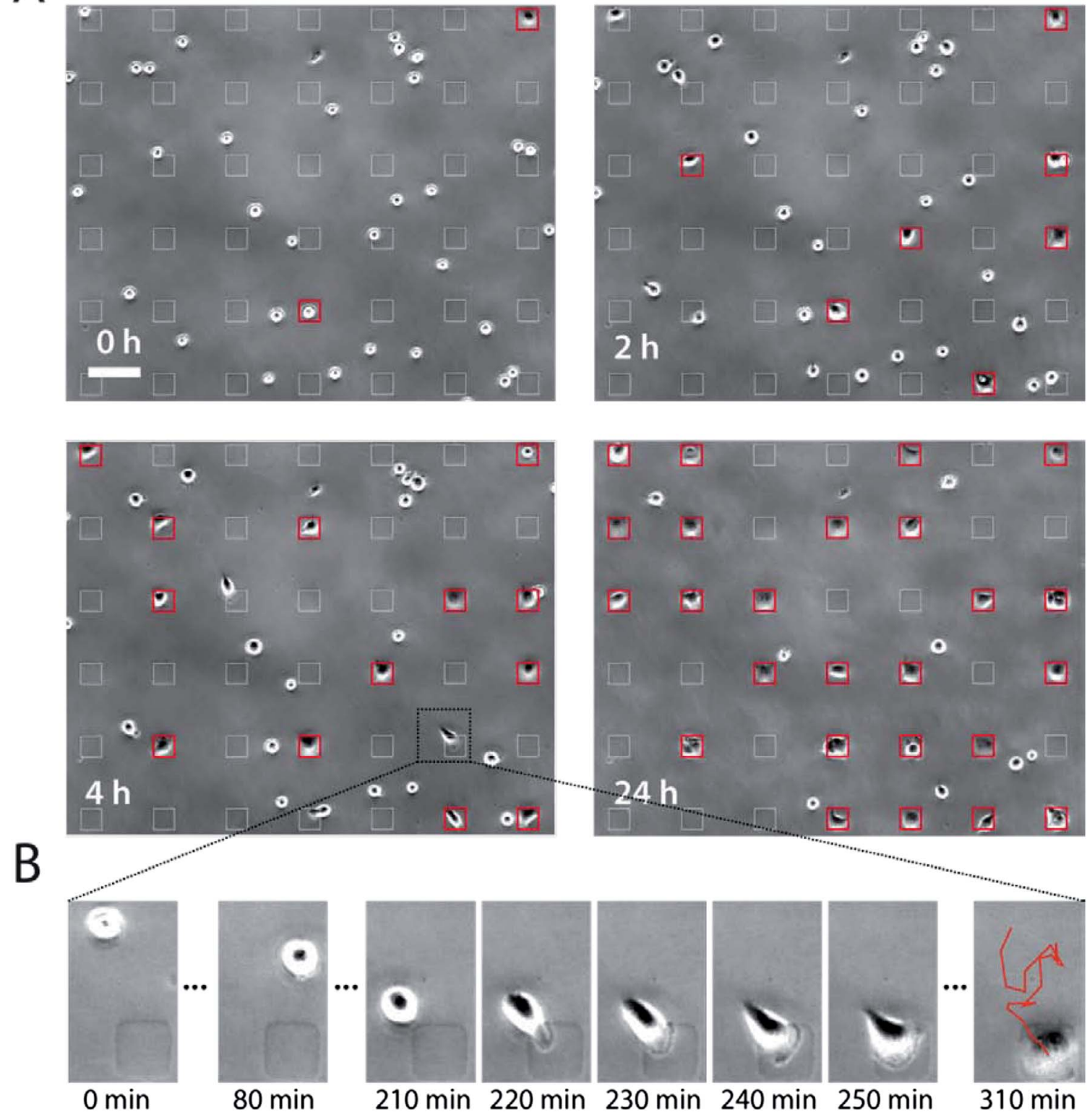

Fig. 2 Dynamics of cellular self-organization. (A) Selected images of the self-organization process during a time-lapse measurement. After cell seeding, cells are randomly distributed. They start to migrate randomly until they find adhesion sites. Gradually, an ordered distribution is obtained. Occupied sites are highlighted in red. Scale bar: $100 \mu \mathrm{m}$ (B). Detailed view of an adhesion process (3× magnification). The red track represents the trajectory of the cell.

Our second defect parameter, $M(t)$, describes the case of multiple occupancies, which is defined as the ratio of multiply occupied sites $N_{\mathrm{MOC}}$ to the total number of occupied sites $N_{\mathrm{OC}}$ : $M(t)=N_{\mathrm{MOC}} / N_{\mathrm{OC}}$. No clear dependency of $N_{\mathrm{MOC}}$ on the lattice distance was observed (Fig. 3D). For all lattice sizes, $N_{\text {MOC }}$ is rather low, ranging from 10 to $20 \%$. For $d_{1}=70 \mu \mathrm{m}$, a slight decrease over time can be observed. For this short lattice distance, cells are most probably able to explore their environment with their filopodia. Thus, the cells can more easily find and invade empty neighbouring sites, which reduces multiple occupancies. After $24 \mathrm{~h}, N_{\mathrm{MOC}}$ can be expected to increase strongly due to cell proliferation.

\section{Universal cell behaviour of self-organization}

To demonstrate that the self-organization process is a generic behaviour of different cell types, we evaluated the aforementioned order parameters for two further cell lines: the hepatocellular carcinoma cell line $\mathrm{HuH} 7$ and the mammary-gland cancer cell line MDA-MB-436. As the spreading areas for these cell lines are basically comparable, the same sizes for the square adhesion sites are used. The phenomenon of cellular selforganization remains unchanged (Fig. S3†). However, as these cells have different properties with regard to adhesion strength, motility and viability, differences in the order parameters were 
A
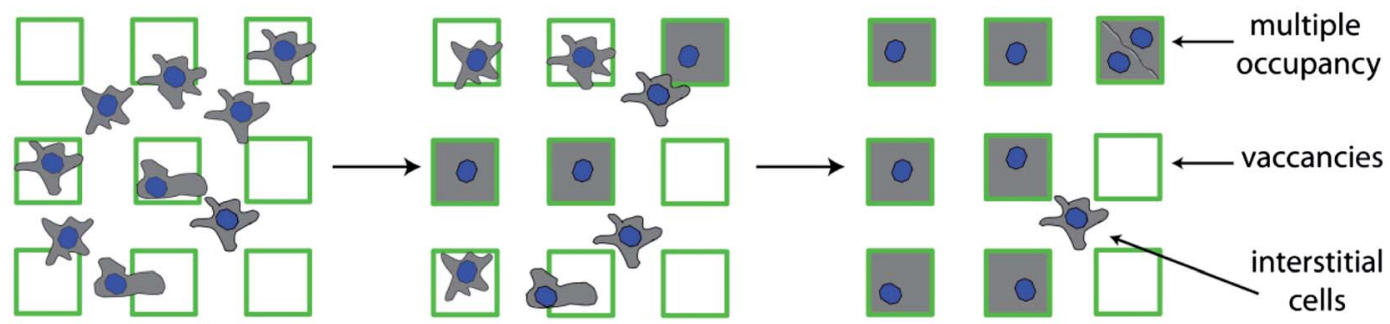

B

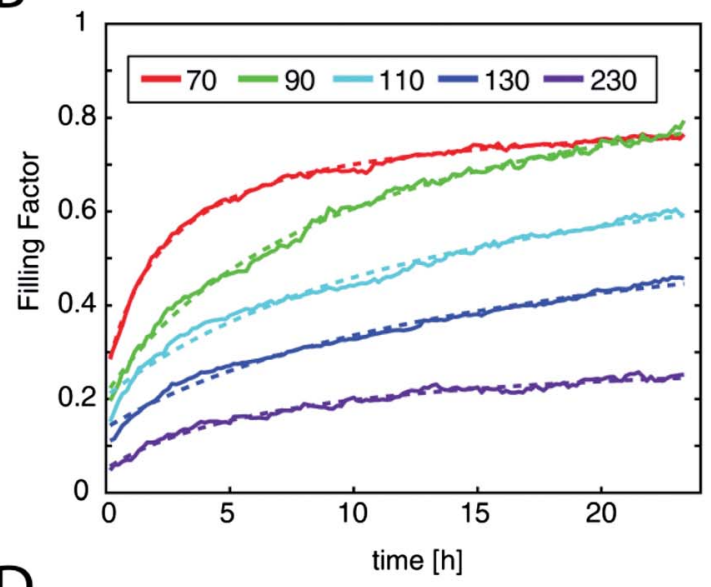

C

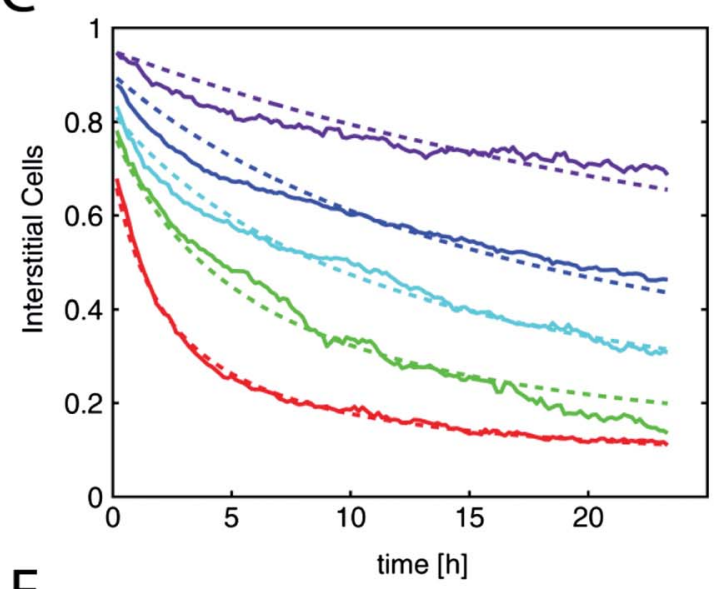

D

$\mathrm{E}$
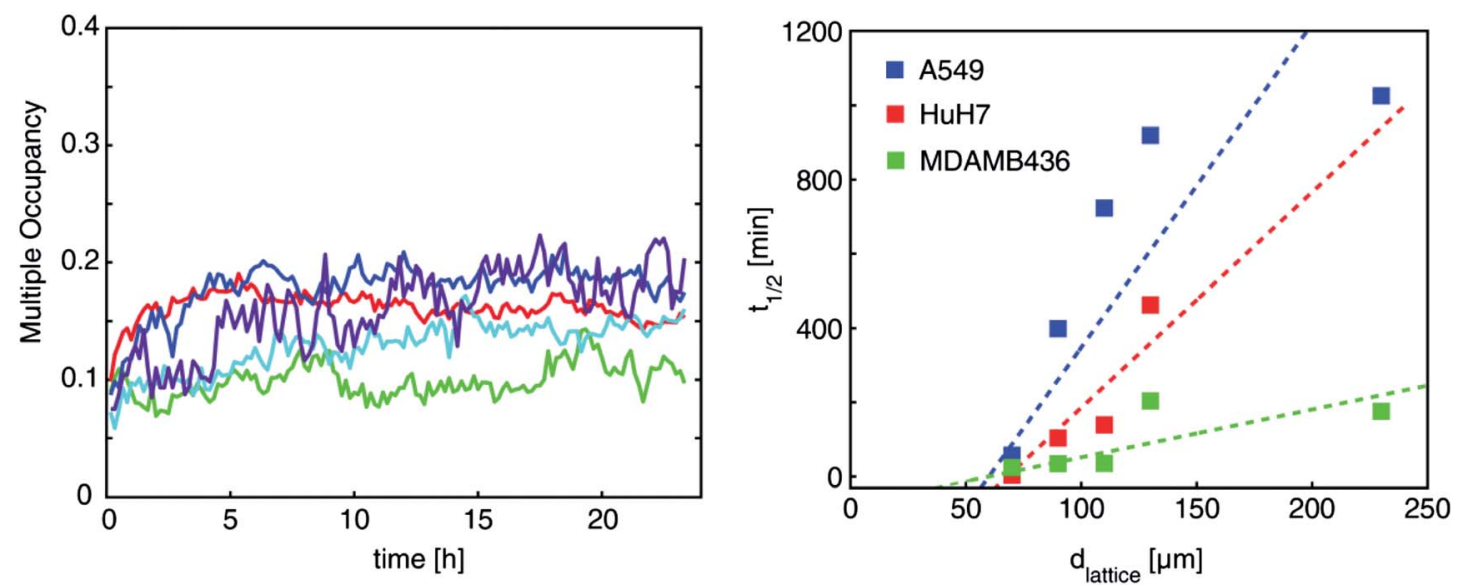

Fig. 3 Dependence of order parameters on lattice spacing. (A) During the self-organization process, optimal filling is compromised by defects such as multiple occupancies, vacancies and interstitial cells. Occupancy is quantified via order parameters: (B) the filling factor describes the ratio of occupied lattice sites to the total number of sites. For A549 the highest filling of $80 \%$ for a lattice distance of $d_{1}=90 \mu \mathrm{m}$ is obtained. (C) The parameter interstitial cells denote the ratio of misplaced to total cells. Misplaced cells after $24 \mathrm{~h}$ are either dead cells or cells which have not yet found a site. (D) The ratio of multiply occupied sites to total occupied sites is denoted by the multiple occupancy parameter. No clear correlation with the lattice distance was observed. (E) Times for half-maximal filling ( $\mathrm{t}_{1 / 2}$, eqn (4)) are plotted against the lattice distance for three different cell lines (A549, HuH7, and MDA-MB-436) with dashed guidelines for the eye. MDA-MB-436 cells showed the smallest $t_{1 / 2}$ values.

measured. HuH7 showed the lowest initial number of interstitial cells (Fig. S3B $\dagger$ ). This can be explained by faster adhesion and spreading to the surface compared to the other cell lines. In Fig. $\mathrm{S} 4, \dagger$ the distribution of the initial distance covered by motile cells is illustrated after $50 \mathrm{~min}$. On average, HuH7 cells have migrated further than A549, although both exhibit similar cell motilities. Thus, HuH7 must start to migrate earlier, and this can be attributed to their faster adhesion. Apart from this, HuH7 behaved very similarly to A549. For MDA-MB-436, the overall filling was lower than for A549 (Fig. S3D $\dagger$ ). A lattice distance of $70 \mu \mathrm{m}$ was too small for MDA-MB-436, as cells tended to form bridges adhering to two sites simultaneously (Fig. S1B $\dagger$ ). Small distances also favoured constant cell migration from one site to another. A larger number of defects (interstitial cells) was observed for MDA-MB-436, and may be attributable to their lower viability on PEG surfaces (Fig. S3E $\dagger$ ). Furthermore, a higher incidence of multiple occupancies was found for MDA-MB-436 from the beginning of the experiment 
(Fig. S3F $\dagger$ ). This observation can be explained by the fact that these cells could not be trypsinized, but were scraped from plates prior to seeding, and thus were not individualized as efficiently, resulting in only $73 \%$ separated single cells $v s$. $90 \%$ for A549 (Fig. S5†). To compare the different cell line's filling rates, the time courses of the filling factors $F(t)$ were fitted to the following phenomenological expression:

$$
F(t)=F_{0}+\frac{F_{\max }}{1+t_{1 / 2} / t}
$$

The offset $F_{0}$ quantifies the sites already occupied at $t=0$. The coefficient of $F_{\max }$ describes the lattice-dependent saturation value of the filling factor. $t_{1 / 2}$ denotes the time at which $F$ reaches half of $F_{\max }$. The fitted $t_{1 / 2}$ values for all lattice distances are plotted in Fig. 3E. The data points show a clear tendency towards increasing filling rates as a function of increasing lattice spacing $d_{1}$. The shortest $t_{1 / 2}$ values are obtained for MDAMB-436, which is consistent with their high motility.

To assess if cell size differences have an influence on filling, cell spreading on the PLL- $g$-PEG surface was analysed (Fig. S6 $\dagger$ ). The major diameter rather than the spreading area was taken into account, as it better reflects the reaching distance of elongated cells. In comparison to a fibronectin-coated surface the major diameter is decreased twofold for A549 and HuH7 and nearly fourfold for MDA-MB-436. Since the adhesion spots are limited, all cell types can only occupy comparably small and similar areas. Thus, the cell specific spreading area has no impact on the filling of the lattice.

\section{Mean time to settle}

Since the self-organization process is a generic phenomenon that depends on the lattice spacing and cell motility, we set out to find a simple model that reveals the time-to-order on a cell chip. How do cells find a lattice site? We adapted the model of chemoreception from Berg and Purcell, ${ }^{\mathbf{3 0}, 31}$ which describes the time needed for a ligand to find a cell surface receptor. Once ligands are attached to the surface of a cell they are assumed to diffuse freely within a membrane until they encounter receptors that are modelled as circular patches. In our case, cells migrate in a random walk on the micro-structured surface $A_{\mathrm{IMG}}$, the field of view of the camera, until they contact adhesion sites of length $s$ (Fig. 4A). We treat our square adhesion sites as circular shapes and employ the analytical expression $\left(N s^{2} \ll A_{\text {IMG }}\right)$ for the meantime to capture derived by Berg and Purcell:
A

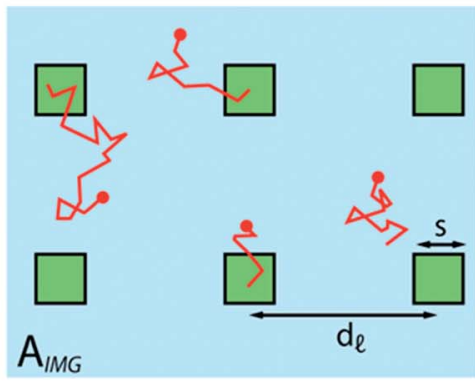

D

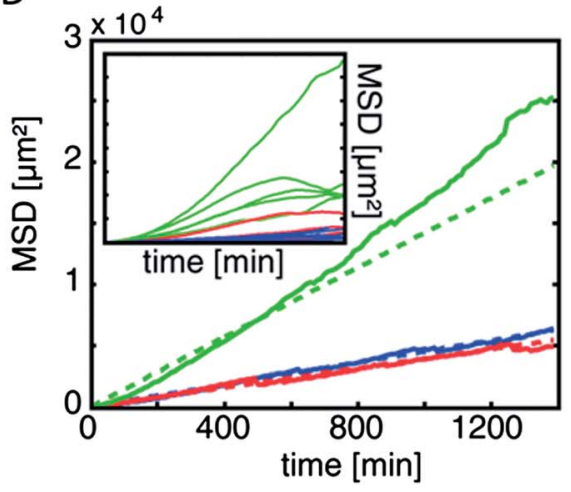

B

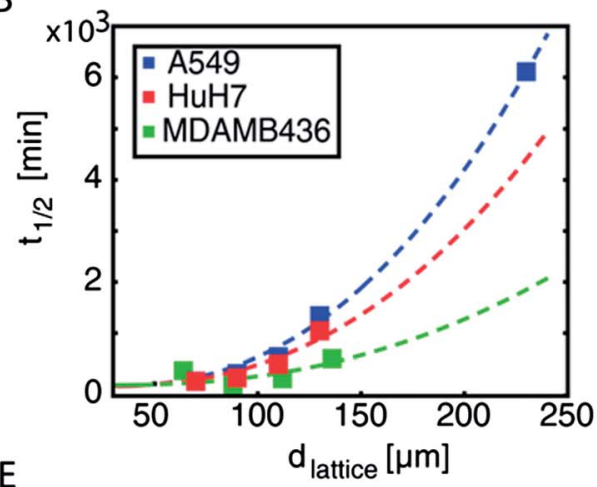

E

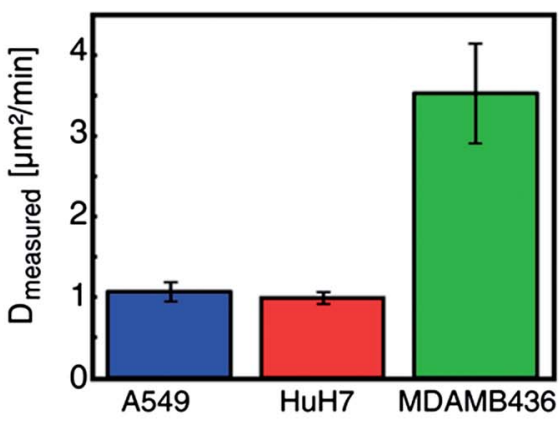

C

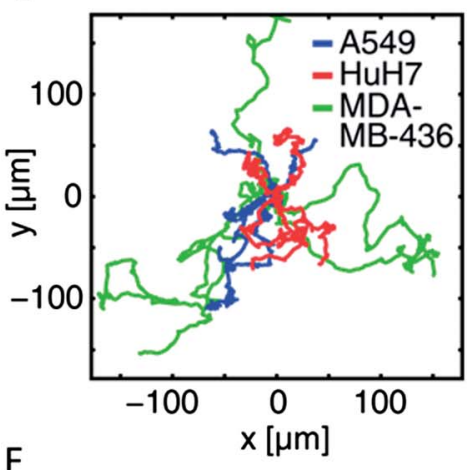

$\mathrm{F}$

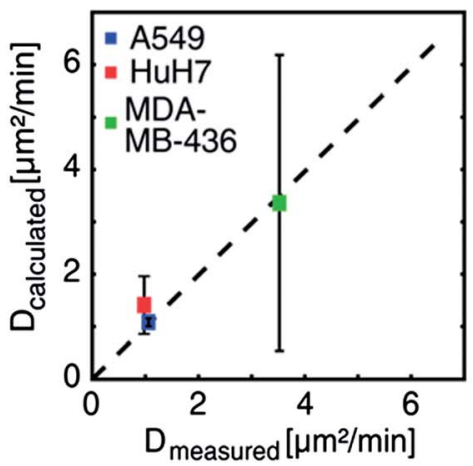

Fig. 4 Mean time to settle. (A) On an image area $A_{I M G}$ (light blue), adhesion sites of size $s$ (green) are distributed in a rectangular lattice with spacing $d_{1}$. The search process for single cells is depicted by the red trajectories. (B) The best fit $t_{1 / 2}$ values obtained by fitting the decay curves for the parameter interstitial cells to eqn (6) are plotted as a function of spacing $d_{1}$. The cell settling process as described by a model adapted from Berg and Purcell (eqn (5)) is in good agreement with these data (dashed lines). (C) Representative trajectories for cells of all three lines migrating on non-patterned PLL-g-PEG are shown. (D) Inset: MSDs of the trajectories in (C) are plotted against time. The averaged MSDs of 100 cell tracks per cell line can be described by a 2D random walk. (E) Fitting $D_{\text {measured }}$ reveals motilities of $1.1 \pm 0.1 \mu \mathrm{m}^{2} \mathrm{~min}^{-1}(\mathrm{~A} 549), 1.0 \pm 0.05 \mu \mathrm{m}^{2} \mathrm{~min}^{-1}$

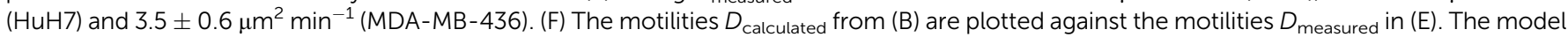
is in good agreement with the measured motilities (correlation of 0.987), error bars correspond to $95 \%$ confidence limits of the fit in (B). 


$$
\overline{t_{1 / 2}\left(d_{1}\right)}=\frac{1.1 A_{\mathrm{IMG}}}{8 \pi N\left(d_{1}\right) D} \ln \left(\frac{1.2 A_{\mathrm{IMG}}}{4 N\left(d_{1}\right) s^{2}}\right)
$$

To compare experiment and theory, the $t_{1 / 2}$ values were derived from the experimentally measured, time-dependent decay curves for the interstitial cell number (Fig. 3C, S3B and $\mathrm{E} \dagger$ ):

$$
I(t)=I_{0}+\frac{I_{0}-I_{\min }\left(d_{1}=70\right)}{1+t_{1 / 2} / t}
$$

$I_{0}$ is the offset at $t=0$. To compare the $t_{1 / 2}$ values for all lattice distances, the same ratio of interstitial cells, $I_{\min }$ of $d_{1}=$ $70 \mu \mathrm{m}$, was assumed, neglecting different saturation levels due to cell death. In Fig. $4 \mathrm{~B}$, the $t_{1 / 2}$ values, obtained by the fits to eqn (6), are plotted as a function of the lattice spacing. The model is found to be in good agreement with the data.

To check that the motilities evaluated from the model are consistent with the measured values, single cell tracks were recorded on PLL- $g$-PEG coated surfaces without patterns. The mean square displacements (MSD) were calculated assuming diffusive behaviour, neglecting persistent cell motion or biased random walk w2-34 $^{32}$ Fig. 4D). Eqn (2) yielded motilities of $1.1 \pm$ $0.1 \mu \mathrm{m}^{2} \mathrm{~min}^{-1}$ for A549, $1.0 \pm 0.05 \mu \mathrm{m}^{2} \mathrm{~min}^{-1}$ for HuH7 and 3.5 $\pm 0.6 \mu \mathrm{m}^{2} \mathrm{~min}^{-1}$ for MDA-MB-436 (Fig. 4E). For all cell lines, these values are in excellent agreement with the motilities derived from the model adapted from Berg and Purcell (Fig. 4F). This result could be expected since we assumed diffusive behaviour of the cells in the first place, but it nevertheless demonstrates that our model allows one to calculate the mean time to order, $t_{1 / 2}$ for any given lattice distance using a single parameter, the motility value for the cell line of interest.

\section{Optimal lattice geometry}

Having modelled the settling of cells as a diffusive search process, we can finally address which lattice geometry is optimal. Taking all order parameters into account, the best filling can be achieved for a geometry with $d_{1}=90 \mu \mathrm{m}$. Here, a single-cell occupancy level of $20 \%$ at the beginning, $45 \%$ after $5 \mathrm{~h}$ and $75 \%$ after $24 \mathrm{~h}$ was obtained for A549 - a 3.5-fold increase in filling rate over $24 \mathrm{~h}$. Similar values were measured for $\mathrm{HuH7}$, with an initial occupancy of $31 \%$, rising to $54 \%$ at $5 \mathrm{~h}$ and $59 \%$ after $24 \mathrm{~h}$. The initial occupancy for MDA-MB-436 was $24 \%$, rising to $40 \%$ after $5 \mathrm{~h}$ and $42 \%$ at $24 \mathrm{~h}$. For both cell lines, the final filling rates are approximately twice as high as the initial ones. Based on the time course of occupation, it should be possible to reduce the incubation time to $5 \mathrm{~h}$ and still obtain reasonable filling. A shorter incubation period is desirable because it prolongs the available time window for single-cell measurements before cell division occurs. This is especially important for cells with high proliferation rates.

Given the motility of a certain cell line, an initial estimate of the best lattice spacing can be made. However, individual cell properties that can complicate the picture must also be taken into account in order to optimize the cell ordering process. These factors include the strength of cell adhesion, viability and cell area on the PEG surface. For example, the largest occupancy and fastest filling for highly motile cells like MDA-MB-436 is obtained for $d_{1}=70 \mu \mathrm{m}$, albeit at the cost of more defects. These cells tend to form bridges between two adhesion sites and are in constant movement. In addition, the transfer of cells between neighbouring sites is favoured for small distances. Conversely, if the lattice distance becomes too large, self-ordering becomes inefficient, as filling time as well as cell death increases. Thus, to obtain a single cell array via self-organization under reasonable conditions, the lattice distance has to be chosen in an appropriate range.

\section{Conclusion}

In comparison to common procedures used to create single-cell arrays, we have shown here that seeding by cellular self-organization offers several advantages. All input cells can be analysed, as this method avoids a washing step after seeding. The cellular self-organization procedure is especially useful for cell types, e.g. rare stem cells, that are available only in limited numbers. ${ }^{35,36}$ As an example, in preliminary experiments we have also observed the self-organising process with mesenchymal stem cells (data not shown). Since a washing step might selectively influence the heterogeneity of surface adhesion, ${ }^{37-39}$ our assay can also be applied to weakly adherent cells that need more time to settle down. Having to wait several hours for selforganization might seem to be incompatible with highthroughput studies, but commonly used approaches also require 2 to $15 \mathrm{~h}$ to allow cells to adhere on the substrate. ${ }^{13,40}$

We presented a theoretical model that describes the generic cell ordering behaviour. The occupation of a regular lattice of adhesion sites follows a random-walk search process. The timeto-order describes the characteristic time-scale needed for a randomly seeded population of cells to arrange themselves on the predefined lattice. High-throughput, single-cell time-lapse analysis requires chips on which isolated cells are positioned at defined sites to facilitate automated image analysis. Hence, a high level of site occupancy and a small number of displaced cells must be achieved after short incubation. For optimal single-cell filling, small distances should be preferred because of faster filling rates. In principle the model allows for optimization of the time-to-order for a specific cell type by rescaling the distances between the adhesion sites, taking the measured cell motility into account.

Beyond that, we reported on several phenomena, such as bridging and multiple occupancies, that limit the densities of adhesion sites that can be achieved in practice. The short-scaled behaviours are interesting in their own rights in terms of cell-tocell communication and open opportunities for further studies in the future. We also showed that various cell lines are capable of self-ordering in our system. Hence, we believe that cellular self-organization is a robust procedure and is applicable in a variety of cell-based assays.

\section{Acknowledgements}

Financial support by the Deutsche Forschungsgemeinschaft (DFG) via project B1 within the SFB 1032, the Excellence Cluster 
'Nanosystems Initiative Munich (NIM)', the Center for NanoScience (CeNS), FP7 EU grants NanoTransKinetics and NanoMILE is gratefully acknowledged. We would like to thank Susanne Seidel and Paul Hardy for critical reading and correcting the manuscript.

\section{Notes and references}

1 D. Muzzey and A. van Oudenaarden, Annu. Rev. Cell Dev. Biol., 2009, 25, 301-327.

2 D. D. Carlo and L. P. Lee, Anal. Chem., 2006, 78, 7918-7925.

3 M. P. Lutolf, P. M. Gilbert and H. M. Blau, Nature, 2009, 462, 433-441.

4 M. Halter, A. Tona, K. Bhadriraju, A. L. Plant and J. T. Elliott, Cytometry, Part A, 2007, 71, 827-834.

5 M. Théry, J. Cell Sci., 2010, 123, 4201-4213.

6 T. Eißing, F. Allgöwer and E. Bullinger, IEE Proc.: Syst. Biol., 2005, 152, 221.

7 M. L. Yarmush and K. R. King, Annu. Rev. Biomed. Eng., 2009, 11, 235-257.

8 M. A. Walling and J. R. E. Shepard, Chem. Soc. Rev., 2011, 40, 4049-4076.

9 G. M. Whitesides, E. Ostuni, S. Takayama, X. Jiang and D. E. Ingber, Annu. Rev. Biomed. Eng., 2001, 3, 335-373.

10 G. Csucs, R. Michel, J. W. Lussi, M. Textor and G. Danuser, Biomaterials, 2003, 24, 1713-1720.

11 C. G. Rolli, H. Nakayama, K. Yamaguchi, J. P. Spatz, R. Kemkemer and J. Nakanishi, Biomaterials, 2012, 33, 2409-2418.

12 A. Azioune, M. Storch, M. Bornens, M. Thery and M. Piel, Lab Chip, 2009, 9, 1640-1642.

13 Y. V. Pan, T. C. McDevitt, T. K. Kim, D. Leach-Scampavia, P. S. Stayton, D. D. Denton and B. D. Ratner, Plasmas Polym., 2002, 7, 171-183.

14 J. Fink, M. Thery, A. Azioune, R. Dupont, F. Chatelain, M. Bornens and M. Piel, Lab Chip, 2007, 7, 672-680.

15 C. P. Jen, J. H. Hsiao and N. A. Maslov, Sensors, 2012, 12, 347358.

16 J. R. Rettig and A. Folch, Anal. Chem., 2005, 77, 5628-5634.

17 K. Chung, C. A. Rivet, M. L. Kemp and H. Lu, Anal. Chem., 2011, 83, 7044-7052.

18 H. Yin, X. Zhang, N. Pattrick, N. Klauke, H. C. Cordingley, S. J. Haswell and J. M. Cooper, Anal. Chem., 2007, 79, 7139-7144.

19 H. Lu, L. Y. Koo, W. M. Wang, D. A. Lauffenburger, L. G. Griffith and K. F. Jensen, Anal. Chem., 2004, 76, 5257-5264.
20 A. Yusof, H. Keegan, C. D. Spillane, O. M. Sheils, C. M. Martin, J. J. O'Leary, R. Zengerle and P. Koltay, Lab Chip, 2011, 11, 2447-2454.

21 A. R. Liberski, J. T. Delaney and U. S. Schubert, ACS Comb. Sci., 2010, 13, 190-195.

22 D. J. Odde and M. J. Renn, Biotechnol. Bioeng., 2000, 67, 312318.

23 D. J. Odde and M. J. Renn, Trends Biotechnol., 1999, 17, 385389.

24 B. R. Ringeisen, C. M. Othon, J. A. Barron, D. Young and B. J. Spargo, Biotechnol. J., 2006, 1, 930-948.

25 K. Ino, M. Okochi, N. Konishi, M. Nakatochi, R. Imai, M. Shikida, A. Ito and H. Honda, Lab Chip, 2008, 8, 134142.

26 S. Gobaa, S. Hoehnel, M. Roccio, A. Negro, S. Kobel and M. P. Lutolf, Nat. Methods, 2011, 8, 949-955.

27 S. Kobel and M. Lutolf, BioTechniques, 2010, 48, ix-xxii.

28 L.-I. Lin, S.-h. Chao and D. R. Meldrum, PLoS One, 2009, 4, 15.

29 S. Youssef, S. Gude and J. O. Rädler, Integr. Biol., 2011, 3, 1095-1101.

30 H. C. Berg and E. M. Purcell, Biophys. J., 1977, 20, 193-219.

31 G. Adam and M. Delbrück, ed. A. Rich and N. Davidson, Reduction of dimensionality in biological diffusion processes in Structural chemistiy and molecular biology, W.H. Freeman \& Company, San Francisco, Calif., 1968, pp. 198-215.

32 A. A. Potdar, J. Lu, J. Jeon, A. M. Weaver and P. T. Cummings, Ann. Biomed. Eng., 2009, 37, 230-245.

33 E. A. Codling, M. J. Plank and S. Benhamou, J. R. Soc. Interface, 2008, 5, 813-834.

34 M. H. Gail and C. W. Boone, Biophys. J., 1970, 10, 980-993.

35 S. Roy, P. Gascard, N. Dumont, J. Zhao, D. Pan, S. Petrie, M. Margeta and T. D. Tlsty, Proc. Natl. Acad. Sci. U. S. A., 2013, 110, 4598-4603.

36 G. Shi, W. Cui, M. Benchimol, Y. T. Liu, R. F. Mattrey, R. Mukthavaram, S. Kesari, S. C. Esener and D. Simberg, PLoS One, 2013, 8, 1-9.

37 A. E. van Merode, H. C. van der Mei, H. J. Busscher and B. P. Krom, J. Bacteriol., 2006, 188, 2421-2426.

38 J. Y. Park, M. A. Arnaout and V. Gupta, J. Biomol. Screening, 2007, 12, 406-417.

39 A. Saterbak, S. C. Kuo and D. A. Lauffenburger, Biophys. J., 1993, 65, 243-252.

40 J. Nakanishi, Y. Kikuchi, T. Takarada, H. Nakayama, K. Yamaguchi and M. Maeda, Anal. Chim. Acta, 2006, 578, 100-104. 